\title{
Advances in targeted therapy: safety of biological agents
}

\section{E C Keystone}

Ann Rheum Dis 2003;62(Suppl II):ii34-ii36

T umour necrosis factor (TNF) antagonists have set a new therapeutic standard for rheumatoid arthritis (RA). The agents including infliximab (Remicade), etanercept (Enbrel), and adalimumab (Humira) have been shown to substantially improve the signs and symptoms, disability, and quality of life while significantly inhibiting joint damage in early and longstanding RA. As with any agent, safety issues in concert with efficacy determine a risk/benefit ratio and hence a position in the therapeutic algorithm. With TNF antagonists key safety considerations include $(a)$ infection, both common and opportunistic; $(b)$ cytopenias; $(c)$ demyelinating disease; (d) lupus-like syndromes; (e) congestive heart failure (CHF) and malignancies, particularly lymphomas. In March 2003 the United States Federal Drug Administration (FDA) convened a meeting to update safety issues related to TNF antagonists, specifically focusing on solid tumours and lymphomas. Safety data from controlled clinical trials were presented for the three TNF antagonists with post-marketing safety data for etanercept and infliximab. All the data are available on the FDA website. The current review will focus on infection, CHF, and malignancy. Much of the information in this review was obtained from the FDA, Arthritis Adviser Committee, the National Databank for Rheumatic Disease (NDRD) of Dr Fred Wolfe, from data on file with Abbott Pharmaceuticals, Amgen Incorporated, and Centocor Incorporated.

Safety data may be obtained from a variety of sources, including placebo controlled, randomised clinical trials, post-approval databases, particularly the USA FDA MedWatch spontaneous reporting programme, and long term registries such as the NDRD of Fred Wolfe as well as registries in Sweden and Germany and England.

A number of factors significantly influence the interpretation of adverse event data. Within clinical trials issues of ascertainment bias in enrolling patients into trials, population homogeneity, lower comorbidities, restricted concomitant drugs, short trial duration, and a relatively small sample size may have substantial influences on the safety profile.

Post-approval (post-marketing) adverse event reporting although influenced to a far lesser extent by population homogeneity, comorbidities, restricted concomitant drugs, duration of follow up, and sample size, still has significant limitations. Post-approval data are limited by substantial

\begin{tabular}{llll}
\hline Table 1 & \multicolumn{3}{l}{ Clinical trials/post-approval } \\
\hline & Etanercept & Infliximab & Adalimumab \\
\hline $\begin{array}{l}\text { RA trials (No of patients) } \\
\text { RA trials } \\
\text { (patient-years exposure) }\end{array}$ & $3839^{*}$ & $1298 \dagger$ & 2468 \\
$\begin{array}{c}\text { Post-approval } \\
\text { (number of patientsts) }\end{array}$ & $>150000$ & 198234 & $\mathrm{NA}$ \\
$\begin{array}{c}\text { Post-approval } \\
\text { (patient-years exposure) }\end{array}$ & $>230000$ & 227559 & $\mathrm{NA}$ \\
\hline
\end{tabular}

*Includes psoriatic arthritis, juvenile rheumatoid arthritis; †ncludes Aspire. underreporting, incomplete and unverifiable data acquisition, and ascertainment bias, particularly with spontaneous reporting such as that in the US FDA MedWatch programme. It has been estimated that $<1 \%$ of serious adverse events are reported to the FDA. ${ }^{1-4}$ The seriousness of the event also influences the propensity for reporting. Another factor affecting reporting is the duration that the drug has been on the market because the number of events reported occur early, particularly within the first two years. Unexpected adverse events are also more likely to be reported.

Interpretation of post-approval adverse event data also depends on how those data are ascertained and reported. For example, for etanercept, data on drug exposure are readily obtained through the prescription numbers, whereas for infliximab difficulty in ascertainment arises as a consequence of bulk shipments to providers and weight based dosing. As a result, patient-year exposure with etanercept is expressed as the length of time for which the drug has been taken, whereas infliximab exposure is expressed as the time which has elapsed since initiation of the drug, regardless of whether it is continued or not. Constituents of the database also influence reporting rates. Thus, for etanercept, juvenile arthritis and psoriatic arthritis data are often included, whereas data on Crohn's disease are often included with infliximab (table 1). Intensity of surveillance will also influence the number of events reported to the company and hence FDA. Finally, the availability of a drug also has an effect on the adverse event profile. Thus only infliximab is approved for funding in America for patients over 65 years of age. This elderly population will have a higher rate of tuberculosis, heart failure, and lymphoma.

The characteristics of the TNF antagonists currently approved differ, particularly in their structure, binding target, half life, capability of in vitro cell lysis, dosing, and efficacy in Crohn's disease (table 2). Whether in vitro cell lysis accounts for differential efficacy in Crohn's disease of TNF producing cells and incidence of granulomatous infections such as Mycobacterium tuberculosis remains unclear. Recent data suggest that lymphocyte apoptosis in the lamina propria of patients with Crohn's disease influences its efficacy. ${ }^{5-7}$

\section{INFECTION}

One of the most important safety issues confronting TNF antagonists is infection. A higher rate of upper respiratory infections have been observed with TNF antagonists than with placebo in clinical trials, although the rate of serious infections was comparable. $M$ tuberculosis has been the most common granulomatous infection observed with TNF antagonists. Its incidence is influenced by age, low socioeconomic status and, particularly, geography. Geography has influenced

Abbreviations: $\mathrm{CHF}$, congestive heart failure; FDA, Federal Drug Administration; NDRD, National Databank for Rheumatic Disease; RA, rheumatoid arthritis; SEER, Surveillance, Epidemiology and End Results (database); SIR, standardised incidence rate; TNF, tumour necrosis factor 
Table 2 TNF antagonists characteristics

\begin{tabular}{llll}
\hline & Infliximab & Etanercept & Adalimumab \\
\hline Structure & Chimeric mAb & TNF IgG 1 & Human mAb \\
& & Fusion protein & \\
Binding target & TNF & TNF, lymphotoxin & TNF \\
Binding affinity & $1.8 \times 10^{9}$ & $10^{10}$ & $2.3 \times 10^{10}$ \\
Half life (days) & $8-9.5$ & $4-5$ & $12-14$ \\
In vitro cell lysis & + & - & + \\
Dose & Every 60 days & Every 3-4 days & Every 7-14 days \\
Efficacy/Crohn's disease & + & - & $?$ \\
\hline
\end{tabular}

the rate of $M$ tuberculosis seen in early clinical trials with adalimumab. All eight of the initial 542 patients treated who developed $M$ tuberculosis were from Germany. Only five cases were seen in 1900 patients treated subsequently, of which two were $M$ tuberculosis positive on screening. The substantial reduction in $M$ tuberculosis reflects screening and likely dose reduction. A similar reduction in $M$ tuberculosis reporting was observed with an $M$ tuberculosis education/screening programme with infliximab.

A recent review of $M$ tuberculosis in patients receiving infliximab in the NDRD showed an increased rate with infliximab relative to patients within the databank not receiving infliximab and a healthy US population (Wolfe F et al, unpublished data). However, patients receiving infliximab were not initially screened for $M$ tuberculosis.

The prevalence of $M$ tuberculosis from post-approval surveillance reflects to a large extent the country of exposure/origin of the patients (table 3). This issue has considerable relevance because $36 \%$ of infliximab use post-approval compared with $10 \%$ of etanercept use occurred in Europe/Norway where the prevalence of $M$ tuberculosis is considerably higher than in the USA. The onset of $M$ tuberculosis after exposure differs significantly, with a median time of onset of 11.2 months with etanercept, whereas $97 \%$ of patients treated with infliximab who developed $M$ tuberculosis did so within seven months. All TNF antagonists have been associated with extrapulmonary $M$ tuberculosis, reflecting the potency of these agents to modulate the immune system. This finding suggests that $M$ tuberculosis screening is prudent with all TNF antagonists. One caveat about skin testing is the incidence of anergy in RA. Several studies have suggested that up to one third of patients with RA demonstrate anergy. ${ }^{8}$ Moreover, it is not uncommon for the $M$ tuberculosis skin test to be placed or read inappropriately, particularly by untrained personnel. For this reason, screening has reduced but not completely

Table 3 Post-approval. M tuberculosis in patients with RA treated with TNF antagonists

\begin{tabular}{lll}
\hline & Etanercept & Infliximab \\
\hline No treated & $>150000$ & 198235 \\
Patient-years exposure & $>230000$ & 227559 \\
Use (\%) & & \\
USA & 90 & 64 \\
EU/Norway & 10 & 36 \\
M tuberculosis reports & 38 & 172 \\
Geography & & \\
$\quad$ USA & 20 & 55 \\
$\quad$ Outside USA & 18 & 117 \\
Characteristics: & & \\
Time to onset & Median 11.2 mo & By 6 infusions: $75 \%$ \\
Miscellaneous (\%) & & By 7 months: $97 \%$ \\
$\quad$ Extrapulmonary & 34 & $30-45$ \\
$\quad$ Miliary & 16 & \\
\hline
\end{tabular}

eliminated $M$ tuberculosis with TNF antagonists. On this account there must be a high degree of awareness for $M$ tuberculosis and other granulomatous diseases. A number of opportunistic infections have been reported with TNF antagonists both within clinical trials and after approval. The most common of these relatively rare infections include histoplasmosis, Pneumocystis carnii, listeriosis, and aspergillosis. Geographical location has relevance here because opportunistic infections are observed more commonly in Europe than in the USA.

\section{CONGESTIVE HEART FAILURE}

Preliminary data suggested that inhibition of TNF ameliorated CHF. However, studies of both etanercept and infliximab failed to demonstrate such a benefit. Two clinical trials of etanercept in CHF involving some 2000 patients with New York Heart Classification (NYHC) functional class 2 to 4 disclosed the possibility of increased mortality from CHF in patients receiving etanercept, particularly in those with less severe heart disease receiving the drug three times a week. Despite these data, it is unclear whether etanercept is associated with increased mortality or morbidity in patients with CHF. Given a relative risk of increased morbidity of 1.2 with etanercept, caution should be used in patients with a history of CHF. Infliximab was evaluated in a 52 week study of 15 patients with NYHC functional class 3 to 4 . An increased rate of hospitalisation and mortality was observed in patients receiving infliximab at a dose of $10 \mathrm{mg} / \mathrm{kg}$ given initially at two and six weeks. No clinical trials of adalimumab in CHF have been carried out. A review of CHF and TNF antagonists etanercept and infliximab in the NDRD, showed no increase in CHF relative to controls (Wolfe F, Michaud MS, unpublished data).

A recent post-approval surveillance report of the treatment of CHF with TNF antagonists showed 47 patients with CHF, of whom 38 had new onset CHF and 9 had exacerbations. ${ }^{9}$ Half of the patients with new onset CHF had no risk factors. Of those developing CHF, 29 patients were receiving etanercept and 18 patients infliximab. The median time of onset from initiation of TNF antagonists to CHF was 3.5 months (range: two hours to two years). Ten patients ( six infliximab and four etanercept treated patients) under the age of 50 developed new onset CHF, three of whom had risk factors for CHF. In nine of 10 patients, CHF improved or resolved with association of treatment.

\section{MALIGNANCY}

The development of malignancy is an issue, given the immunosuppressive nature of the TNF antagonists. For solid tumours, data with etanercept and infliximab demonstrated that the number of tumours seen during follow up of patients treated in clinical trials was comparable with the cohort matched for age, sex, and race obtained from the Surveillance, Epidemiology and End Results (SEER) database of the National Cancer Institute of the USA. 
Table 4 Lymphoma with TNF antagonists

\begin{tabular}{|c|c|c|c|c|c|}
\hline Study & Country & $\begin{array}{l}\text { Patients with } \\
\text { RA (n) }\end{array}$ & $\begin{array}{l}\text { Follow up } \\
\text { (years) }\end{array}$ & $\begin{array}{l}\text { SIR for } \\
\text { cancer }\end{array}$ & $\begin{array}{l}\text { SIR for } \\
\text { lymphomas }\end{array}$ \\
\hline Gridley (1993) ${ }^{1}$ & Sweden & 11683 & 20 & 1.0 & 2.4 \\
\hline Mellenkjaer $(1996)^{2}$ & Denmark & 20699 & 14 & 1.1 & 2.4 \\
\hline Isomaki $(1978)^{3}$ & Finland & 46101 & 7 & 1.1 & 2.7 \\
\hline Matteson $(1991)^{5}$ & Canada & 530 & 7 & 1.5 & 8.0 \\
\hline Baecklund (1998) ${ }^{4}$ & Sweden & 11683 & 18 & - & 1.0 (L act) \\
\hline & & & & & 5.4 (Mact) \\
\hline & & & & & 25.8 (Hact) \\
\hline Ekstrom $(2003)^{6}$ & Sweden & 76527 & $<1-20+$ & 1.09 & 2.0 \\
\hline \multicolumn{6}{|c|}{$\begin{array}{l}\text { 1. Gridley G, et al. J Natl Cancer Inst 1993;85:307-11. } \\
\text { 2. Mellenkiaer L, et al. Eur J Cancer 1996;32A:1753-7. } \\
\text { 3. Isomaki, et al. J Chron Dis 1978;31:691-6. } \\
\text { 4. Baecklund E, et al. BM 1998;317:180-1. } \\
\text { 5. Matteson E, et al. J Rheumatol 1991;18:809-14. } \\
\text { 6. Ekstrom K, et al. Arthritis Rheum 2003:8;963-70. }\end{array}$} \\
\hline
\end{tabular}

Table 5 Lymphoma with TNF antagonists

\begin{tabular}{llll}
\hline & Etanercept & Infliximab & Adalimumab \\
\hline Treated/exposure: & & & \\
$\quad$ No of patients & 3389 & $1298^{*}$ & 2468 \\
$\quad$ Patient-years & $(8336)$ & $(2458)$ & $(4870)$ \\
Total No & $6 \rightarrow 9$ & 4 & 10 \\
Hodgkin's/non-Hodgkin's $\dagger$ & $3 / 6$ & $1 / 3$ & $1 / 9$ \\
Mean time to onset (months), range & $21(0.1-4.6)$ & $10-19(6.4)$ & $18(2.0-42.0)$ \\
SIR lymphoma (95\% conf. interval) & $2.31 \rightarrow 3.47(1.59$ to 6.59) & $6.4(1.7$ to 16.3) & $5.4(2.6$ to 10.0) \\
\hline * Rheumatoid arthritis only; tmajority diffuse large B cells. & &
\end{tabular}

The risk of lymphoma, however, is more complex given that the incidence of lymphomas is increased in patients with RA. The standardised incidence rate (SIR) - that is, ratio of the observed to the expected number of lymphomas within the SEER database has been reported to be as high as 8.0 (table 4). ${ }^{10}$ This risk has been shown to correlate with activity and severity of disease as well as exposure to immunosuppressive agents. In clinical trials, SIRs observed for lymphomas with TNF antagonists are within the range of previous reports (table 5). Most of the lymphomas associated with the use of TNF antagonists are non-Hodgkin's lymphomas with a mean time to onset averaging 10-21 months. Data from the NDRD showed modest increases in the SIRs relative to patients receiving methotrexate alone and those not receiving disease modifying drugs, with wide and overlapping 95\% confidence intervals (Wolfe F, Michaud MS, unpublished data). Postapproval surveillance reports of lymphoma with TNF antagonists showed reporting rates of $\sim 0.02-0.03$ events/100 patients-years, whereas the expected rate of lymphomas in a normal population aged 65 within the SEER database is 0.07 events/100 patient-years. The actual incidence post-approval is unclear because the degree of underreporting of lymphomas is difficult to ascertain.

Taken together, the data to date suggest that there is a higher rate of lymphomas in patients receiving TNF antagonists than in normal subjects. Whether the risk of lymphomas is higher with TNF antagonists than in patients receiving conventional disease modifying drugs remains unclear.

\section{CONCLUSION}

As with any immunosuppressive agent, safety considerations remain an issue. For the most part, the serious adverse events are uncommon and the risks appear manageable. Despite the risks inherent in treatment with TNF antagonists the risk/benefit ratio to date is extremely good. However, only prolonged observational studies will deal with the long term incidence of known adverse events as well as early ascertainment of rare events.

\section{ACKNOWLEDGEMENTS}

I thank Jeannette Henderson for her excellent secretarial assistance. I also thank Abbott Incorporated, Amgen Incorporated, and Centocor Incorporated for their assistance in providing their data on file, Drs A Kavanaugh and J Cush for their assistance in the preparation of this report and Dr Fred Wolfe who provided data from the National Databank for Rheumatic Disease (NDRD).

\section{Author's affiliation}

E C Keystone, Rebecca MacDonald Centre for Arthritis and Autoimmune Disease, Mount Sinai Hospital, The Joseph and Wolf Lebovic Building, 2nd Floor, Room 2-006, 60 Murray Street, Toronto, Ontario M5G 1X',

Canada; Ksnow@mtsinai.on.ca

\section{REFERENCES}

1 Scott H, Rosenbaum, S, Waters W, et al. Rhode Island physicians recognition and reporting of adverse drug reactions. R I Med J 1987;70:311-16.

2 Rawlins MD. Pharmcovigilance: paradise lost, regained or postponed. J R Coll Physicians Lond 1995;29:41-9.

3 Martin RM, Kapoor KV, Wilton LV, et al. Underreporting of suspected adverse drug reactions to newly marketed ("black triangle") drugs in general practice: observational study. BM 1998;317:119-20.

4 Heeley E, Riley J, Layton D, et al. Prescription event monitoring and reporting of adverse drug reactions. Lancet 2001;358:1872-3.

5 Len Hove T, van Monffrans C, Peppelenbosch MP, et al. Infliximab treatment induces apoptosis of lamina propria T lymphocytes in Crohn's disease. Gut 2002;50:206-11.

6 Lugering A, Schmidt $M$, Lugering $N$, et al. Infliximab induces apoptosis in monocytes from patients with Crohn's disease by using a caspase-dependent pathway. Gastroenterology 2001: 121; 1145-1 157.

7 van den Brande JMHV, Braat, $\mathrm{H}$; van den Brink, GR et al Infliximab but not etanercept induces apoptosis in lamina propria T-lymphocytes from patients with Crohn's disease. Gastroenterology 2003: 124; 1774-1785.

8 Emery P, Panayi G, Symmons D, et al. Mechanisms of depressed delayed-type hypersensitivity in rheumatoid arthritis: the role of protein energy malnutrition. Ann Rheum Dis 1984:43:430-4.

9 Kavon HJ, Cole TR, Cuffe MS, et al. Case reports of heart failure after therapy with a tumor necrosis factor antagonist. Ann Intern Med 2003; 138:807-12.

10 Baecklund E, Ekbom A, Sparen P, et al. Disease activity and risk of lymphoma in patients with rheumatoid arthritis. Nested case-control study. BM 1998;317:180-1. 